\title{
Feasibility of a telementoring approach as a practical training for transurethral enucleation of the benign prostatic hyperplasia using bipolar energy: a pilot study
}

\author{
Marco Amato ${ }^{1,2} \cdot$ Ahmed Eissa $^{1,3} \cdot$ Stefano Puliatti ${ }^{1,2,4} \cdot$ Cristian Secchi $^{5} \cdot$ Federica Ferraguti $^{5} \cdot$ Marco Minelli $^{5}$. \\ Agostino Meneghini ${ }^{6}$. Isotta Landi ${ }^{7}$ Giulio Guarino ${ }^{1}$ - Maria Chiara Sighinolfi ${ }^{1}$ Bernardo Rocco ${ }^{1}$. \\ Giampaolo Bianchi $^{1} \cdot$ Salvatore Micali ${ }^{1}$
}

Received: 26 February 2020 / Accepted: 8 January 2021 / Published online: 4 February 2021

(c) The Author(s), under exclusive licence to Springer-Verlag GmbH, DE part of Springer Nature 2021

\begin{abstract}
Introduction Telementoring is one of the applications of telemedicine capable of bringing highly experienced surgeons to areas lacking expertise. In the current study, we aimed to assess a novel telementoring application during the learning curve of transurethral enucleation of the prostate using bipolar energy (TUEB).

Material and methods A telementoring system was developed by our engineering department. This application was used to mentor ten prospective cases of TUEB performed by an expert endourologist (novice to the TUEB). A questionnaire was filled by the operating surgeon and the mentor to provide subjective evaluation of the telementoring system. Finally, the outcomes of these patients were compared to a control group consisting of ten consecutive patients performed by the mentor. Results Ten consecutive TUEB were performed using this telementoring application. Delayed and interrupted connection were experienced in two and one patients, respectively; however, their effect was minor, and they did not compromise the safety of the procedure. None of the patients required conversion to conventional transurethral resection of the prostate. Only one patient in our series experienced grade IIIb complication.

Conclusion The telementoring application for TUEB is promising. It is a simple and low-cost tool that could be a feasible option to ensure patients' safety during the initial phase of the learning curve without time and locations constraints for both the mentor and the trainee; However, it should be mentioned that telementoring cannot yet replace the traditional surgical training with the mentor and trainee being in the operative room. Further studies are required to confirm the current results
\end{abstract}

Keywords Telementoring $\cdot$ Telemedicine $\cdot$ Learning curve $\cdot$ Benign prostatic hyperplasia

Salvatore Micali

Salvatore.micali@unimore.it

1 Department of Urology, University of Modena and Reggio Emilia, Via del Pozzo, 71, 41124 Modena, Italy

2 ORSI Academy, Melle, Belgium

3 Urology Department, Faculty of Medicine, Tanta University, Tanta, Egypt

4 Department of Urology, Onze Lieve Vrouw Hospital, Aalst, Belgium

5 Department of Sciences and Methods for Engineering (DISMI), University of Modena and Reggio Emilia, Reggio Emilia, Italy

6 Maria Degli Angeli Hospital, Azienda ULSS 5 Polesana, Adria, Italy

7 Department of Psychology and Cognitive Sciences, University of Trento, Royereto, Italy

\section{Introduction}

Surgical training is a fundamental part of all surgical specialties. It started long time ago by the apprenticeship model in its primitive form where a trainee works for the surgeon usually for 5-7 years in return for surgical instructions and to learn from the surgeon [1-3]. Apprenticeship is the origin of Halstedian model of training "see one-do one-teach one", which, is an effective training model but it has been associated with a prolonged training period in order to attain the required surgical skills [4]. In these settings, the reduction of the available training hours (for both trainees and trainers), the increased cost of training, the rapid technological advancements in the field of telecommunication and surgical instrumentation, and the steep learning curve of certain surgical procedures resulted in 
an urgent need for an effective training alternatives [5, 6]. Telemedicine is one of the innovations that gained great interest since 1950s and it is defined as the use of the telecommunication technologies to provide health care services and information (live video-audio interaction between physicians and/or patients) [7]. Furthermore, its value has increased during the current COVID-19 pandemic [8]. Telementoring describes a situation when, an expert surgeon guides a less experienced surgeon through advanced or novel cases from a remote location [9]. The first telementoring experience dates back to 1997, when, Rosser et al. [10], reported the feasibility of telementoring for advanced training of laparoscopic colectomy.

In the field of urology, new technologies are being rapidly adopted and the nature of practice is changing as well as the definition and requirements of competency in urology. Thus, urologists were among the first surgeons to adopt telementoring with five urological cases (including laparoscopic radical nephrectomy) being telementored between the USA and Italy [11], and two cases (laparoscopic radical nephrectomy and laparoscopic varicocelectomy) telementored between USA and Singapore [12]. Furthermore, there has been great interest in the use of telementoring in robotic-assisted and endoscopic urologic surgeries [5, 13].

Laser enucleation of the prostate has shown to be a promising size-independent alternative to transurethral resection of the prostate (TURP) for the management of benign prostatic hyperplasia (BPH); however, it was limited to specific centers as a result of its steep learning curve and higher initial cost [14-16]. Transurethral enucleation of the prostate using bipolar energy (TUEB) appeared as a lower cost alternative for management of large-sized benign prostatic hyperplasia as it can be done using the standard bipolar electrosurgical unit and a modified electrode [17]. However, it is also associated with a steep learning curve, which may extend up to 100 cases [18]. Xiong et al. [19], reported that the conversion rates to standard TURP significantly decreased after 30 cases, while, the efficiency of enucleation significantly increased following 50 cases. In these settings, we aimed to assess the feasibility of telementoring for endoscopic enucleation of the prostate with a novel, easy-to-use, low-cost, and easily accessible telementoring application.

\section{Material and methods}

\section{Study type and population}

This is a prospective study carried out at the Department of Urology, University of Modena and Reggio Emilia, between January 2019 and June 2019. The study was approved by the local ethical committee. Ten consecutive patients suffering from benign prostatic hyperplasia (BPH) associated with moderate-to-severe lower urinary tract symptoms (LUTS), identified by the international prostate symptom score (IPSS), were enrolled in the current study (group A). Dual medical therapy with alpha-blockers and 5-alpha reductase inhibitors were tried in all patients before referral for surgery. All the patients with previous history of transurethral prostatic surgery, neurogenic bladder, prostate or bladder cancer, urethral stricture, abnormal digital rectal examination, and abnormal coagulation profile were excluded from the current study. On the other hand, a group of ten patients who had undergone TUEB by the mentor, during the same period of time, were included as a control group (group B).

\section{Informed consent}

There is no standardized informed consent for telementored patients. The issue of patients' privacy also represents a significant concern for the clinical implementation of telementoring. However, a model created ad hoc was approved by the ethical committee. Each patient had an individual meeting with the surgeon and the mentor (video conference with the mentor) to adequately discuss about the security techniques used for protection of patient's privacy. Patients are informed that the operation would have continued in the classical form if there were connection problems. All patients agreed to undergo the procedure. Privacy has been preserved thanks to a secure connection and video recording of the surgical field only.

\section{Variables}

\section{Preoperative variables}

All patients underwent a preoperative uroflowmetry, transrectal ultrasound (for assessment of prostate size), and flexible cystoscopy. Furthermore, the preoperative IPSS was calculated for all patients. Patients with elevated prostate-specific antigen and/or suspicious digital rectal examination underwent prostate biopsy. Furthermore, the preoperative hemoglobin was also evaluated.

\section{Surgical technique and variables}

All the cases were performed according to the technique described by Hirasawa [18]; however we used ultrasoundguided morcellation after the adenoma was removed [20]. Blood loss and intraoperative complications were also recorded. Furthermore, the quality of the connection (delayed or interrupted transmission) was assessed. 


\section{Postoperative variables}

The duration of catheterization, length of hospital stay, uroflowmetry, and IPSS at 3 months, were evaluated. Complications are reported and graded according to the Clavien-Dindo classification [21].

\section{Telementoring application}

A web application was developed as a result of the collaboration between Engineering Department and the Department of Urology in the University of Modena and Reggio Emilia. This application is capable of providing real-time video streaming of the endoscopic camera view, audio communication link between the mentor and the surgeon, and offering the ability to draw illustrations on the surgeon's monitor for remote guidance (telestrating). The entire system is composed of a PC in the operating room, Bluetooth headset, video converter device, and a workstation for the mentor (PC or tablet). The connection used was encrypted wlan. $720 \mathrm{p}$ video at $25 \mathrm{fps}$. The video has always been recorded for a possible postoperative teaching. Figure 1 shows the monitor screen with the telestration function as seen by the surgeon.

\section{Telementoring}

Both the surgeon (from Modena, Italy) and the mentor (from Rovigo, Italy) are experienced endourologists. Before starting the telementored procedures, the surgeon observed different procedures performed by the mentor to become familiar with the technique of prostate enucleation. The mentor has performed more than 1000 operations with this technique. At the end of each procedure, a questionnaire (specially developed for the assessment of this application) was given to the operating surgeon (trainee) regarding the aspects of the intervention, the network connection and the training impact. This questionnaire aimed to subjectively evaluate the safety, efficacy, learning, and connection quality using a score ranging from 0 to $6(0=$ not at all; $6=$ perfect); subsequently, an overall score combining the scores of these four sections is given (maximum 24 points). A second questionnaire (maximum 16 points) was filled by the mentor only to stratify the procedure (prostate volume, ASA score, complexity), evaluate the setup of the operating room (instrument verification, connection verification), and the execution of various phases of the intervention with a score from 0 to $4(0=$ surgical technique switch, 4 execution in total autonomy). Finally, the mentor was asked to give a subjective evaluation for the hemostasis (maximum 12 points) and the morcellation techniques of the surgeon

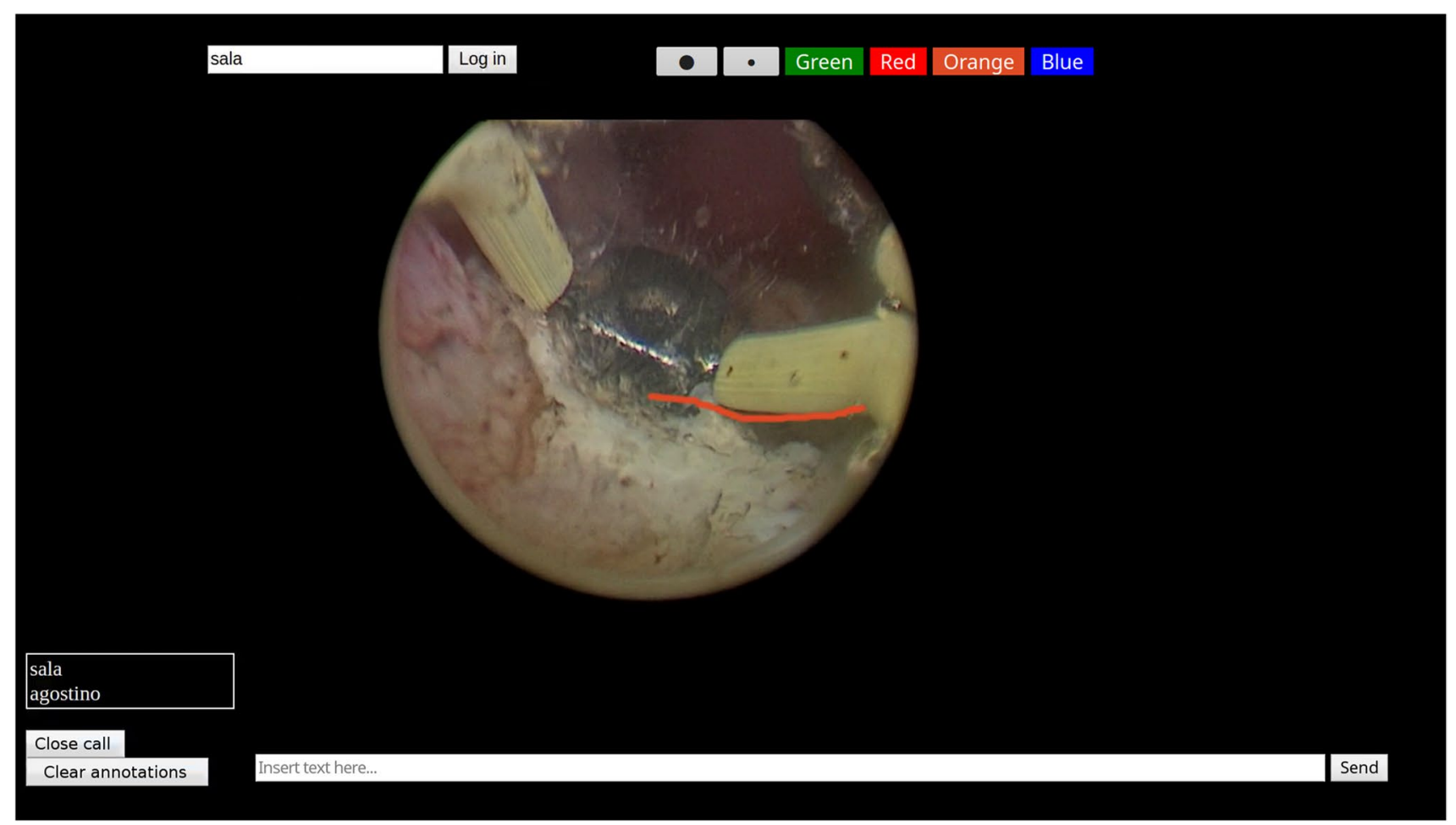

Fig. 1 Telestration: the mentor draws on the screen to guide the surgeon 
(maximum 4 points). A copy of the questionnaire is found in the supplementary material.

\section{Statistical analysis}

The recommendations for sample size in pilot studies are variable in literature; however, according to Isaac and Michael [22], sample sizes between 10 and 30 patients are sufficient for testing hypothesis, but may overestimate weak treatment effects. Furthermore, Treece E.W. and Treece J.W. [23], supported the same sample size $(N=10)$ for piloting an instrument. In this setting, ten patients were included in each of our study groups. On the other hand, Shapiro-Wilk test and Kolmogorov-Smirnov tests were used to assess the distribution of variables. All continuous variables are reported as mean and standard deviation in case of normal distribution, while in case of non-normally distributed variable data are presented as median and interquartile range. Similarly, the categorical variables are presented as percentages. Paired Student's $t$-test or Wilcoxon signed-rank test was used to compare pre- and post-operative variables (Qmax, IPSS, and hemoglobin), while independent Student's $t$-test or the Mann-Whitney $U$ test was used to compare between both groups. $P$ value of $<0.05$ was considered significant.

\section{Results}

\section{Bipolar enucleation results}

Overall, 20 patients were included in the current study, of which, ten consecutive patients in the surgeon's institute were prospectively enrolled in the telementoring group (group A), and the data of ten consecutive patients were retrospectively collected from the mentor's institute to form the control group (group B). The mean age of the patients was $69 \pm 8.6$ years (range $49-77$ years) and $68.9 \pm 10.3$ years (range $54-89$ years) in group A and B, respectively. The median size of the prostate was $50 \mathrm{cc}$ (range 30-120 cc) in group A and 78.5 (range 40-120 cc) in group B. Table 1 summarizes the characteristics of the included patients. All the cases were completed without complete or partial conversion to TURP. The median operative time was significantly longer in the group A compared to group B (125 min versus $75 \mathrm{~min}, p=0.009)$. Similarly, the catheterization and the hospitalization times were significantly shorter in group B compared to group A, but this may be explained by the hospital protocol which indicates that all patients are discharged after catheter removal (Table 1). There was no significant difference as regards the complications between both groups $(p=1.00)$. In the telementoring group (group A), all patients were continent at the time of catheter removal except for one patient $(10 \%)$ who suffered from incontinence that was managed with pharmacological therapy (Clavien-Dindo II). Furthermore, one patient (10\%) developed urethral stenosis that was discovered during a cystoscopy performed for the evaluation of a bladder mass 6 months following the TUEP (Clavien-Dindo IIIb) and was managed by optical urethrotomy. On the other hand, in the control group, only one patient suffered from hematuria (Clavien-Dindo II) and another patient demonstrated acute urinary retention (Clavien-Dindo II) (Table 2). Both patients in the control group were managed conservatively without the need for any surgical intervention. All the patients showed significant improvement of the IPSS (25 versus $11, p=0.008$, and 23.5 versus $4.5, p=0.005)$ and the $Q \max (9$ versus $18, p=0.018$, and 6 versus $26, p=0.005$ ) in group A and $\mathrm{B}$, respectively. On the other hand, there was a statistically significant drop of the hemoglobin (13.77 versus 12.90 ,
Table 1 Summary of patients' characteristics, preoperative and postoperative data

\begin{tabular}{lllllr}
\hline & $\begin{array}{l}\text { Group A } \\
\text { Mean } \pm \text { SD }\end{array}$ & Range & Group B & Range & $p$ value \\
\hline Age (years) & $69 \pm 8.6$ & $49-77$ & $68.9 \pm 10.3$ & $54-89$ & 0.963 \\
Prostate size (cc)* & $50(21.3)$ & $30-120$ & $78.5(37.5)$ & $40-120$ & $\mathbf{0 . 0 1 9}$ \\
Preoperative Qmax (ml/s)* & $9(4)$ & $6-11$ & $6(5)$ & $5-11$ & 0.274 \\
Preoperative IPSS* & $25(8)$ & $18-30$ & $23.5(7.8)$ & $20-31$ & 1.000 \\
Preoperative hemoglobin (g/dl) & $13.8 \pm 1.7$ & $11.3-17$ & $14.2 \pm 0.6$ & $13.3-15.1$ & 0.464 \\
Postoperative Qmax (ml/s)* & $18(5)$ & $15-24$ & $26(2.3)$ & $24-28$ & $<\mathbf{0 . 0 0 1}$ \\
Postoperative IPSS * & $11(3)$ & $6-27$ & $4.5(1.25)$ & $3-6$ & $<\mathbf{0 . 0 0 1}$ \\
Postoperative hemoglobin (g/dl) & $12.9 \pm 1.7$ & $10.9-15$ & $13.2 \pm 0.7$ & $12.1-14.3$ & 0.656 \\
Operative time (min)* & $125(75)$ & $70-240$ & $75(36.3)$ & $60-135$ & $\mathbf{0 . 0 0 9}$ \\
Catheter time (days)* & $3(2.5)$ & $2-6$ & $1(0.25)$ & $1-2$ & $<\mathbf{0 . 0 0 1}$ \\
Length of hospital stay (days)* & $4(2.5)$ & $3-7$ & $2(0.3)$ & $2-3$ & $<\mathbf{0 . 0 0 1}$ \\
\hline
\end{tabular}

Bold values are statistically significant (alpha $<0.05$ )

*Median and IQR are used 
$p=0.04$ in group A and 14.19 versus $13.16, p<0.001$ in group B).

\section{Telementoring results}

All the included patients in group A were completed without major problems in the telementoring application. During the transmission, the mean network speed was $85 \mathrm{MB} / \mathrm{s}$. The telestrator functioned normally in all the 10 patients allowing the mentor to give instructions to the trainee. Delayed transmission (few milliseconds) was experienced in only two patients without any effect on the patient's safety or the course of the procedure. Interruption of the connection occurred in only one patient; however, the connection was restored rapidly and the procedure was completed normally, in the remaining patients there was no image buffering or pixilation.

As regards the results of the postoperative questionnaire, the surgeon and the mentor reported that the safety, efficacy, learning, and connection quality were $70 \%$, $100 \%, 100 \%$, and $60 \%$, respectively. The mean overall score for the first part of the questionnaire was $23 \pm 1.49$ (range 20-24). Considering the mentor's evaluation for the various phases of the procedure, the mean value was $13.40 \pm 1.43$ (range 11-15). Furthermore, the efficacy of hemostasis and morcellation was $100 \%$ as evaluated by the mentor. Overall, the surgeon showed progressive improvement in the quality of surgery from one patient to the other.

\section{Discussion}

Since the introduction of telementoring in 1997 [10], it has shown tremendous improvement in the quality of connection thanks to the advancement in the telecommunication technology. Telementoring is capable of overcoming the

Table 2 Summary of the complications and connection problems in the telementored patients

\begin{tabular}{lll}
\hline & Complication & Number \\
\hline Telemen- & Bipolar enucleation complications & \\
toring & Incontinence (Clavien-Dindo II) & 1 \\
patients & Urethral stenosis (Clavien-Dindo IIIb) & 1 \\
& Telementoring and connection problems & \\
& Delayed transmission & 2 \\
& Interrupted connection & 1 \\
Control & Control group & \\
& Hematuria (Clavien-Dindo II) & 1 \\
& Acute urinary retention (Clavien-Dindo II) & 1 \\
\hline
\end{tabular}

geographical limitation of mentoring and brings highly experienced mentors to areas lacking expertise, thus allowing inexperienced surgeons to perform procedures that otherwise would not be attempted due to the complexity of the procedure and the lack of surgeon's experience [5, 24]. There has been a great interest in the use of telementoring among different specialties including neurosurgery, ophthalmology, otolaryngology, vascular surgery, and general surgery due to its educational benefits, the reported high satisfaction, and low complication rates (5\%) [25]. In this setting, it allows remote mentors to provide 'real-time' instructions to trainees and gives inexpert surgeons the confidence to perform difficult surgeries [24]. Despite the potential advantages of telementoring, it has not gained widespread use due to the paucity of mentors willing to participate, inadequate video conferencing capabilities, and the lack of uniform medicolegal guidelines and the patient privacy laws across the different parts of the world [26]. Furthermore, the costs of actual telementoring system are high, considering the annual costs for equipment maintenance and software support [27]. However, during the current COVID-19 pandemic this technology might be of great importance as it allows the continuation of the training programs while reducing the personal contact between the trainee and the mentor, thus reducing the risk of infection $[8,28]$.

We firmly believe that 'internet' is the future platform for learning and improvement of surgical skills due to the reduction of the time available for training and the increased cost of training. Furthermore, internet data transfer has grown exponentially, potentially eliminating the connection limits of the past. The fifth generation wireless-network technology has just been tested successfully for telementoring between Barcelona-Spain and Shanghai-China [29]. In this setting, we aimed to assess the feasibility and affordability of teaching an endoscopic technique using an easy-to-use and low-cost application. Our application is based on simple and low-cost instrumentation that are normally present in most of the operating rooms (computer, Bluetooth headset, video convertor device, and a tablet for the mentor). This renders it relatively cheaper compared to the currently commercially available telementoring platforms [30]. Furthermore, the application provides telestration function (illustrations drawn by the mentor on his workstation and displayed on the working monitor of the trainee), which together with the verbal guidance can greatly amplify the potential benefits of telementoring approach [30].

On the other hand, simulators are considered another attractive models for training during the learning curve of enucleation or transurethral resection of the prostate [31-33]. However, it should be noted the actual operative experience is an essential part of the training process, thus, simulation should be used only in conjunction with traditional teaching methods [33]. Furthermore, simulation-based 
training seems to be more expensive compared to our telementoring application [5]. We believe that a combination of simulation and telementoring could significantly facilitate the training of difficult urologic surgery including bipolar enucleation of the prostate.

In the current series, all the cases were performed by a single expert endourologist who was novice to endoscopic enucleation of the prostate and this may explain the significantly longer operative time in our series compared to the cases performed by the experienced surgeon (group B) and to the times reported in the literature $[18,19]$. In a review of more than 1000 cases, Shigemura et al. [34] demonstrated that the increase of surgeons' experience significantly contribute to shorter operative times. Interestingly, the complication rates in the telementoring patients were not significantly different compared to patients in group B, and overall, it was comparable to the rates reported by Xiong et al. [19] during their initial 50 cases. These findings may reflect the safety of the telementoring-based training for difficult endoscopic procedures as TUEB.

Despite the significant improvement in the IPSS and Qmax in both groups, there was a significant difference in the postoperative IPSS and the postoperative Qmax between the telementoring group and the control group, which may be explained by the difference in the huge experience between the trainee and the mentor [34]. Furthermore, this difference may be explained by the difference in the preoperative size of the prostate, where Shang Chen et al. [35] reported that the operation efficiency is increased with the increase in the preoperative size of the prostate.

On the other hand, the questionnaire rendered low results for the safety $(70 \%)$ and connection quality $(60 \%)$, which may be explained by the small number of patients included in our study and by the connection problems we encountered during the first three patients, however, these results had no impact on the overall evaluation of application. Yet, this factor should be interpreted with caution as the poor connection in our series may be overwhelmed by the experience of the trainee in the endourological surgery.

To our knowledge, this is the first study that assesses the use of a telementoring application during the initial phase of the learning curve of the TUEB, yet our study is not devoid of limitations. Firstly, the small sample size in our study is among the main limitations; however, this is just a pilot and feasibility study and further studies are being planned. Furthermore, all the cases in our series were performed by an expert endourologist and we did not include a resident with limited experience in endourology, thus our results must be interpreted with caution as it may apply only to surgeons with great experience in endourology.

\section{Conclusion}

Telementoring is a promising approach for teaching complex urological endoscopic procedures such as TUEB. Our application is characterized by being simple, low cost, and easy to use allowing the mentor to provide instruction to the trainee in a 'real-time' fashion. It could improve the quality of surgical education at a lower cost. However, it should be mentioned that telementoring cannot yet replace the traditional surgical training with the mentor and trainee being in the operative room.

Supplementary Information The online version contains supplementary material available at https://doi.org/10.1007/s00345-021-03594-9.

\section{Compliance with ethical standards}

Conflict of interest The authors declare that there is no conflict of interest regarding the publication of the current study.

\section{References}

1. Franzese CB, Stringer SP (2007) The evolution of surgical training: perspectives on educational models from the past to the future. Otolaryngol Clin North Am 40(1227-35):vii. https://doi. org/10.1016/j.otc.2007.07.004

2. Puliatti S, Mazzone E, Dell'Oglio P (2020) Training in robot-assisted surgery. Curr Opin Urol 30:65-72. https://doi. org/10.1097/MOU.0000000000000687

3. Sherbiny AE, Eissa A, Ghaith A, et al (2018) Training in urological robotic surgery. Future perspectives. Arch Esp Urol 71(1):97-107

4. Shaharan S (2014) Evaluation of surgical training in the era of simulation. World J Gastrointest Endosc 6:436. https://doi. org/10.4253/wjge.v6.i9.436

5. El Sherbiny A, Eissa A, Ghaith A et al (2018) Training in urological robotic surgery. Future perspectives. Arch Esp Urol 71:97

6. Shamim Khan M, Ahmed K, Gavazzi A et al (2013) Development and implementation of centralized simulation training: evaluation of feasibility, acceptability and construct validity. BJU Int 111:518-523. https://doi.org/10.1111/j.1464-410X.2012.11204.x

7. Rosser JC, Gabriel N, Herman B, Murayama M (2001) Telementoring and teleproctoring. World J Surg 25:1438-1448. https://doi. org/10.1007/s00268-001-0129-X

8. Fahmy DH, El-Amawy HS, El-Samongy MA et al (2020) COVID19 and dermatology: a comprehensive guide for dermatologists. J Eur Acad Dermatol Venereol. https://doi.org/10.1111/jdv.16545

9. Raison N, Khan MS, Challacombe B (2015) Telemedicine in surgery: What are the opportunities and hurdles to realising the potential? Curr Urol Rep 16:7

10. Rosser JC, Wood M, Payne JH et al (1997) Telementoring: a practical option in surgical training. Surg Endosc 11:852-855. https:// doi.org/10.1007/s004649900471

11. Micali S, Virgili G, Vannozzi E et al (2000) Feasibility of telementoring between Baltimore (USA) and Rome (Italy): the 
first five cases. J Endourol 14:493-496. https://doi.org/10.1089/ end.2000.14.493

12. Lee BR, Png DJC, Liew L et al (2000) Laparoscopic telesurgery between the United States and Singapore. Ann Acad Med Singap 29:665

13. Castaneda P, Ellimoottil C (2019) Current use of telehealth in urology: a review. World J Urol 38:2377

14. Netsch C, Bach T, Herrmann TRW et al (2013) Evaluation of the learning curve for thulium vapoenucleation of the prostate (ThuVEP) using a mentor-based approach. World J Urol 31:12311238. https://doi.org/10.1007/s00345-012-0894-1

15. Saredi G, Pirola GM, Pacchetti A et al (2015) Evaluation of the learning curve for thulium laser enucleation of the prostate with the aid of a simulator tool but without tutoring: comparison of two surgeons with different levels of endoscopic experience endourology and technology. BMC Urol. https://doi.org/10.1186/s1289 4-015-0045-2

16. Brunckhorst O, Ahmed K, Nehikhare O et al (2015) Evaluation of the learning curve for holmium laser enucleation of the prostate using multiple outcome measures. Urology 86:824-829. https:// doi.org/10.1016/j.urology.2015.07.021

17. Mallikarjuna C, Nayak P, Ghouse SM et al (2018) Transurethral enucleation with bipolar energy for surgical management of benign prostatic hyperplasia: our initial experience. Indian J Urol 34:219-222. https://doi.org/10.4103/iju.IJU_71_16

18. Hirasawa Y, Kato Y, Fujita K (2017) Transurethral enucleation with bipolar for benign prostatic hyperplasia: 2-year outcomes and the learning curve of a single surgeon's experience of 603 consecutive patients. J Endourol 31:679-685. https://doi.org/10.1089/ end.2017.0092

19. Xiong W, Sun M, Ran Q et al (2013) Learning curve for bipolar transurethral enucleation and resection of the prostate in saline for symptomatic benign prostatic hyperplasia: experience in the first 100 consecutive patients. Urol Int 90:68-74. https://doi. org/10.1159/000343235

20. Tzou DT, Metzler IS, Tsai C et al (2019) Ultrasound-guided morcellation during difficult holmium laser enucleation of the prostate. Urology. https://doi.org/10.1016/j.urology.2019.09.027

21. Dindo D, Demartines N, Clavien PA (2004) Classification of surgical complications: a new proposal with evaluation in a cohort of 6336 patients and results of a survey. Ann Surg 240:205-213

22. Isaac S, Michael WB (1995) Handbook in research and evaluation: a collection of principles, methods and strategies useful in the planning, design, and evaluation of studies in education and the behavioral sciences, 3rd edn. EdITS Publishers, New York

23. Treece EW, Treece JW (1982) Elements of research in nursing, 3rd edn. Mosby, St. Louis
24. James HE (2016) Pediatric neurosurgery telemedicine clinics: a model to provide care to geographically underserved areas of the United States and its territories. J Neurosurg Pediatr 25:753-757. https://doi.org/10.3171/2016.6.PEDS16202

25. Augestad KM, Bellika JG, Budrionis A et al (2013) Surgical telementoring in knowledge translation-clinical outcomes and educational benefits: a comprehensive review. Surg Innov 20:273-281. https://doi.org/10.1177/1553350612465793

26. Moses GR, Doarn CR (2008) Barriers to wider adoption of mobile telerobotic surgery: engineering, clinical and business challenges. Stud Health Technol Inform 132:308-312

27. Antoniou SA, Antoniou GA, Franzen J et al (2012) A comprehensive review of telementoring applications in laparoscopic general surgery. Surg Endosc 26:2111-2116. https://doi.org/10.1007/ s00464-012-2175-x

28. Puliatti S, Eissa A, Eissa R et al (2020) COVID-19 and urology: a comprehensive review of the literature. BJU Int. https://doi. org/10.1111/bju.15071

29. Lacy AM, Bravo R, Otero-Piñeiro AM et al (2019) 5G-assisted telementored surgery. Br J Surg 106:1576-1579. https://doi. org/10.1002/bjs. 11364

30. Hung AJ, Chen J, Shah A, Gill IS (2018) Telementoring and telesurgery for minimally invasive procedures. J Urol 199:355-369. https://doi.org/10.1016/j.juro.2017.06.082

31. Childs BS, Manganiello MD, Korets R (2019) Novel education and simulation tools in urologic training. Curr Urol Rep 20:81

32. Kailavasan M, Berridge C, Athanasiadis G et al (2020) Design, implementation, and evaluation of a novel curriculum to teach transurethral resection of the prostate (TURP): a 3-year experience of urology simulation bootcamp course. World J Urol. https ://doi.org/10.1007/s00345-020-03104-3

33. Kuronen-Stewart C, Ahmed K, Aydin A et al (2015) Holmium laser enucleation of the prostate: simulation-based training curriculum and validation. Urology 86:639-646. https://doi. org/10.1016/j.urology.2015.06.008

34. Shigemura K, Yamamichi F, Kitagawa K et al (2017) Does surgeon experience affect operative time, adverse events and continence outcomes in holmium laser enucleation of the prostate? A review of more than 1,000 cases. J Urol 198:663-670. https://doi. org/10.1016/j.juro.2017.04.087

35. Chen SS, Lin MJ, Weng MF et al (2018) Influence of prostate size on the perioperative and postoperative outcome of transurethral plasmakinetic enucleation of the prostate: results of 892 patients with 3 years of follow-up. Kaohsiung J Med Sci 34:576-582. https ://doi.org/10.1016/j.kjms.2018.03.001

Publisher's Note Springer Nature remains neutral with regard to jurisdictional claims in published maps and institutional affiliations. 